\title{
Gait Controllability for Legged Robots
}

\section{Bill Goodwine}

\author{
Aerospace and Mechanical Engineering \\ University of Notre Dame \\ Notre Dame, Indiana 46556
}

\author{
Joel Burdick \\ Mechanical Engineering \\ California Institute of Technology \\ Pasadena, California 91125
}

\begin{abstract}
We present a general method for determining controllability of a class of kinematic legged robots. The method is general in that it is independent of the robot's morphology; in particular, it does not depend upon the number of legs. Our method is based on an extension of a nonlinear controllability test for smooth systems to the legged case, where the relevant mechanics are not smooth. Our extension is based on the realization that legged robot configuration spaces are stratified. The result is illustrated with a simple example.
\end{abstract}

\section{Introduction}

This paper considers the issue of nonlinear gait controllability for legged robots. That is, we consider if a specified gait can allow the robot to move in any direction. This is important for two reasons. First, such controllability is a necessary condition for motion planning algorithms. (Clearly, if the robot cannot move in all directions, it is then impossible to specify an arbitrary path for the robot to follow). Secondly, controllability is a useful design tool.

Generally speaking, for autonomous robots, and legged robots in particular, there is a trade off between the complexity of the robot and the associated sophistication of the controller. In other words, if the robot has many degrees of freedom, it will be relatively simple to devise a control strategy for it; conversely, if the robot has relatively few degrees of freedom, a control strategy which exploits the particular geometry or other nonlinear features of the robot may be necessary.

As a specific example, consider the miniature sixlegged hexapod robot illustrated in Figure 1 (potentially fabricated using MEMS technology). This model will be fully explored in Section 4. Note that each leg has only two degrees of freedom: the robot can only lift its legs up and down and move them forward and backward. As mentioned, such limited control authority may be desirable in practical situations because it decreases the mechanical complexity of the robot. This hexapod requires only 12 degrees of freedom; whereas, a conventional design requires 18 degrees of freedom. However, such decreased complexity comes at the cost of requiring more sophisticated control theory. Note

that for this model, it is not immediately clear whether the robot can move "sideways," and if it cannot move sideways, then it is not controllable.

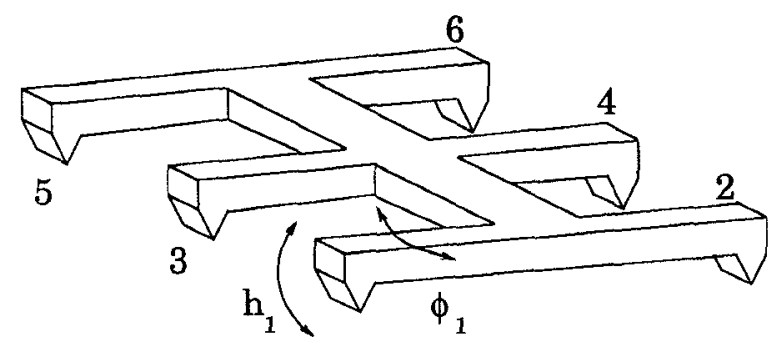

Figure 1. Simple hexapod robot.

There is a vast literature on legged locomotion analysis, control, and motion planning. However, to our knowledge, the issue of controllability has not been extensively analyzed. Most prospective robotic mechanisms have contained sufficiently many degrees of freedom such that controllability is obvious; however, as mentioned, such complexity may be reduced if compensated by sufficiently sophisticated control methodologies. Our approach is to formulate results in sufficient mathematical generality so that it will apply to robotics problems independent of morphology. In contrast, most legged robotics efforts have focused either on a particular morphology (e.g. biped [1], quadruped [2], or hexapod [3]) or a particular locomotion assumption (e.g. quasi-static [3] or hopping [4]). In contrast, previous work by the authors [5], [6] and this paper are general, in that they apply independent of morphology, and additionally apply to problems of a class which include legged robots.

There has been some recent work directed to uncovering principles that span all morphologies so that they are of general applicability. For example, Kelly and Murray [7] showed that a number of "kinematic" locomotive systems can be modeled using connections on principal fiber bundles and also provide results on controllability. Ostrowski [8], [9] developed analogous results for a class of "dynamic" nonholonomic locomotion systems. However, these results assume that the equations of motion for the system are smooth, which prohibits their application to legged robotics problems, 
where the equations of motion are discontinuous. Standard nonlinear controllability tests (Chow's theorem and variations thereof), require that the system's equations of motion be smooth. The main contribution of this work is the extension of these standard nonlinear control methodologies to a class of problems where the equations of motion are discontinuous.

\section{Background}

In this section we introduce Chow's theorem and the notion of a stratified configuration space.

\subsection{Mathematical Preliminaries}

This paper is concerned with driftless robotic control systems with equations of motion of the form

$$
\dot{x}=g_{1}(x) u^{1}+\cdots+g_{m}(x) u^{m},
$$

where $x$ is the state of the robot and is a point in configuration manifold $M$, the $u^{i}$ are the control inputs, and the $g_{i}(x)$ are vector fields defined on $M$. The driftless assumption limits our results to quasi-static robotic locomotion.

Central to nonlinear control theory is the Lie bracket. Given two vector fields, $g_{1}(x)$ and $g_{2}(x)$, their Lie bracket is the product defined by

$$
\left[g_{1}, g_{2}\right]=\frac{\partial g_{2}}{\partial x} g_{1}-\frac{\partial g_{1}}{\partial x} g_{2}
$$

Lie brackets can be thought of as "new directions" in which the system can flow because of the relationship

$$
\phi_{\epsilon}^{-g_{2}} \circ \phi_{\epsilon}^{-g_{1}} \circ \phi_{\epsilon}^{g_{2}} \circ \phi_{\epsilon}^{g_{1}}\left(x_{0}\right)=\phi_{\epsilon^{2}}^{\left[g_{1}, g_{2}\right]}\left(x_{0}\right)+\mathcal{O}\left(\epsilon^{3}\right) \text {, }
$$

where $\phi_{t}^{g}$ denotes the flow along the vector field $g$ for time $t$, i.e., the solution to $\dot{x}=g(x)$. In words, if we appropriately "modulate" the control inputs $u^{1}$ and $u^{2}$, to leading order, the resulting flow is along the Lie bracket, $\left[g_{1}, g_{2}\right]$. The span of a set of vector fields is a distribution, $\Delta=\operatorname{span}\left\{g_{1}, \ldots, g_{m}\right\}$, and its closure under Lie bracketing is the involutive closure of the distribution, denoted $\bar{\Delta}$, which is the smallest distribution such that if $f, g \in \bar{\Delta}$, then $[f, g] \in \bar{\Delta}$.

A system is small time locally controllable (STLC) if it can reach any point in an open neighborhood of its starting point in arbitrarily small time. A fundamental result in nonlinear control theory is Chow's Theorem which relates involutive distributions to controllability.

THEOREM 2.1 Let $\bar{\Delta}$ be the involutive distribution formed by the vector fields in Equation 1. If $\operatorname{dim}(\bar{\Delta})=$ $\operatorname{dim}(M)$, then the system is STLC.

Unfortunately, Chow's theorem can not be applied to analyze legged systerns because the Lie bracket calculations require that the equations of motion be sufficiently differentiable. Our goal is to develop an analog of Chow's theorem for such legged systems.

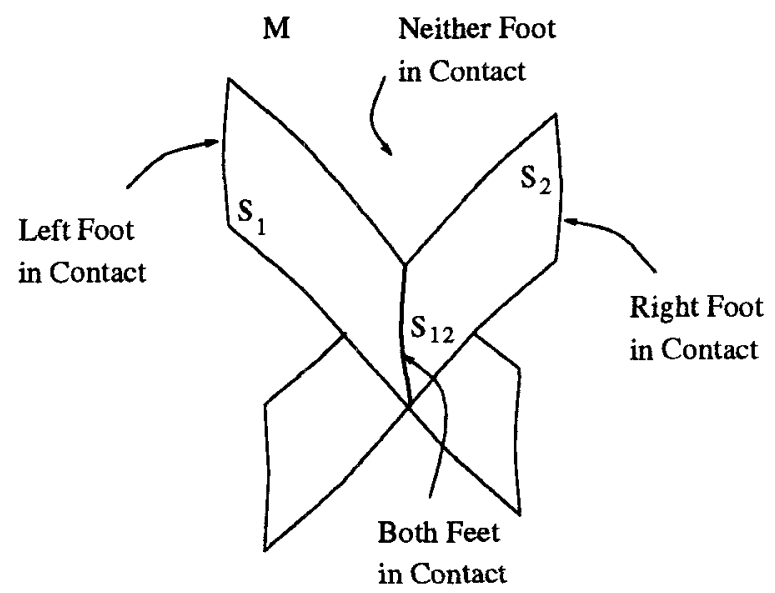

Figure 2. Stratified configuration manifold structure for a biped robot.

\subsection{Stratified Configuration Spaces}

We will motivate our definition of a stratified configuration space with a simple example. Consider a biped robot. The configuration manifold for the robot describes the spatial position and orientation of the robot as well as variables such as joint angles which describe its internal geometry. The set of configurations corresponding to one of the feet in contact with the ground is a codimension one submanifold of the configuration space. The same is true when the other foot contacts the ground. Similarly, when both feet are in contact with the ground, the system is on a codimension 2 submanifold of the configuration space formed by the intersection of the single contact submanifolds. The structure of the configuration manifold for such a biped is abstractly illustrated in Figure 2. The goal in this paper is to exploit the geometric structure of such configuration spaces.

Because the robot is subjected to different constraints on each submanifold described above, it will have different equations of motion depending upon which combination of feet are in contact with the ground. Also, except for when the robot transitions from a state where a foot is off of the ground to one where a foot contacts the ground, the equations of motion for the system are smooth.

We will refer to the configuration space for the biped robot in Figure 2 as stratified. By considering legged robot systems more general than the biped in Figure 2, we can develop a general definition of stratified configuration spaces. Let $M=S_{0}$ denote the legged robot's entire configuration manifold. Let $S_{i} \subset M$ denote the codimension one submanifold of $M$ that corresponds to all configurations where only the $i^{t h}$ foot contacts the terrain. Denote, the intersection of $S_{i}$ and $S_{j}$, by 


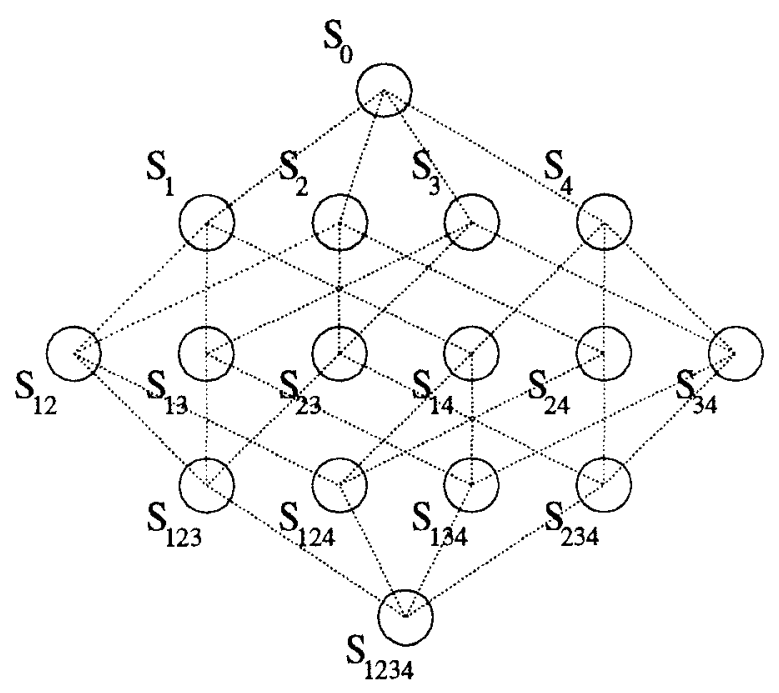

Figure 3. Four Level Stratification

$S_{i j}=S_{i} \cap S_{j}$. The set $S_{i j}$ corresponds to states where both the $i^{\text {th }}$ and $j^{\text {th }}$ feet are on the ground. Further intersections can be similarly defined in a recursive fashion: $S_{i j k}=S_{i} \cap S_{j} \cap S_{k}=S_{i} \cap S_{j k}$, etc. Note that the ordering of the indices is irrelevant, i.e., $S_{i j}=S_{j i}$. We will refer to the submanifolds $S_{i}$, as well as their recursive intersections $S_{i j}, S_{i j k}$, etc, as strata. We will term the lowest dimension stratum containing the point $x$ as the bottom stratum, and any other submanifolds containing $x$ as higher strata. When making relative comparisons among different strata, we will refer to lower dimension strata as lower strata, and higher dimension strata as higher strata. Denote an arbitrary stratum by $S_{I}=S_{i_{1} i_{2} \cdots i_{n}}, I=\left\{i_{1} i_{2} \cdots i_{n}\right\}$, and note that its codimension is $n$, the length of the multi-index subscript.

Figure 3 illustrates a stratification with four levels, which corresponds to the configuration space of a quadruped. In the figure, the nodes of the graph correspond to the different strata. The edges connecting the nodes indicate whether it is possible for the system to move from one stratum to another, i.e., if the nodes are connected by an edge, then the system can move between the strata, if there is no edge, the system cannot move between the strata. While the figure simply illustrates edges between nodes only one level apart, multi-level jumps may be possible, in which case there would be an edge connecting strata that are more than one level apart.

Definition 2.2: (GAIT)

A gait an ordered sequence of strata:

$$
\mathcal{G}=\left\{S_{I_{1}}, S_{I_{2}}, \ldots, S_{I_{n}}, S_{I_{n+1}}=S_{I_{1}}\right\} .
$$

where the first and last element are identical, indicating that the gait is a closed loop. The gait can be considered as a closed path through the graph structure in Figure 3.

In order for the gait to be meaningful, it must be possible for the system to switch from stratum $S_{I_{i}}$ to $S_{I_{i+1}}$ for each $i$. In Figure 3, this corresponds to each stratum $S_{I_{i}}$ in the sequence being connected to $S_{I_{i+1}}$ and $S_{I_{n}}$ being connected to $S_{I_{1}}$. Limitations on gaits, such as stability requirements, could be expressed as limitations on the cyclic gait paths.

Whether a stratum is permissible partly depends upon whether the equations of motion for the system can be expressed as a kinematic system (recall Equation 1), in a neighborhood of the point of interest. For example, for a biped robot, clearly if it lifts both feet off of the ground, it is not a kinematic system because the fact that gravity will make it fall back to the ground. For robotic systems, the possibility of movement from a higher to a lower stratum will be obvious in a given problem, since it will be obvious whether or not, from a given configuration, it is possible for the robot to move a foot to the terrain.

EXAMPLE 2.3 Section 4 investigates the hexapod example from Figure 1 in detail, but here we illustrate one possible gait for it. Assume that the hexapod walked with a tripod gait moving legs 1-4-5 in unison and legs 2-3-6 in unison. This assumption reduces the high dimensional and complex graph structure of the system to a very low dimensional and simple one, as illustrated in Figure 4. In the figure, the arrows show the cyclic path of the gait. It will always be possible for this system to move from a higher stratum $\left(S_{145}\right.$ or $S_{236}$ ) onto the bottom stratum $\left(S_{123456}\right)$, since the robot can always put its feet on the ground regardless of its configuration.

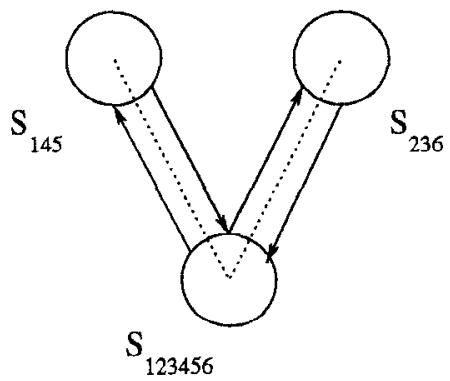

Figure 4. The Simplified Hexapod Graph

Associated with each stratum in a stratified system will be a set of equations of motion for the system. Since we consider driftless nonlinear systems, the equations of motion at $x \in S_{I}$ are expressed as

$$
\dot{x}=g_{I, 1}(x) u^{I, 1}+\cdots+g_{I, n_{I}}(x) u^{I, n_{I}} .
$$


The involutive closure of the distribution defined by the span of these vector fields on $S_{I}$ will be denoted $\bar{\Delta}_{I}$. We assume that the vector fields in the equations of motion for any given stratum are well defined at all points in that stratum, including points contained in any substrata of that stratum. For example, the vector fields $g_{0, i}(x)$ are well defined for $x \in S_{I}$. Note, however, that they do not represent the equations of motion for the system in the substrata, but, nonetheless, are still well defined as vector fields.

Finally, we assume that the only discontinuities present in the equations of motion are due to transitions on and off of strata. These correspond to states where one or more feet make or break ground contact. Specifically, vector fields defined on any stratum are assumed to be smooth when restricted to that stratum. When a configuration manifold is consistent with the above description, we will refer to it as a stratified configuration manifold. Generic quasi-static legged robotic systems have such a structure.

\section{Gait Controllabilitytest footnote}

In a general stratified structure, there will be one bottom stratum, denoted $S_{B}$, defined by the intersection of all the codimension 1 strata in the configuration space. In Figure 3, this is stratum $S_{1234}$ and for the hexapod example, this is stratum $S_{12456}$. For a legged robot, this bottom stratum corresponds to the set of points in the configuration space where all the feet are in contact with the ground.

Given an open set $V \subseteq M$, define $R^{V}\left(x_{0}, T\right)$ to be the set of states $x$ such that there exists $u:[0, T] \rightarrow$ $\mathcal{U}$ that steers the control system from $x(0)=x_{0}$ to $x(T)=x_{f}$ and satisfies $x(t) \in V$ for $0 \leq t \leq T$, where $\mathcal{U}$ is the set of admissible controls. Define

$$
R^{V}\left(x_{0}, \leq T\right)=\bigcup_{0<\tau \leq T} R^{V}\left(x_{0}, \tau\right) .
$$

We will refer to $R^{V}\left(x_{0}, \leq T\right)$ as the set of states reachable up to time $T$. For gait controllability, the set of admissible controls must be consistent with the gait, i.e., admissible control inputs must steer the the system through the sequence of strata that define the gait.

DeFinition $3.1 \mathrm{~A}$ gait, $\mathcal{G}=\left\{S_{I_{1}}, S_{I_{2}}, \ldots, S_{I_{n}}, S_{I_{1}}\right\}$ is gait controllable from $x_{0}$ if the reachable set $R^{V}\left(x_{0}, \leq T\right)$ contains a neighborhood of $x_{0}$ for all neighborhoods $V$ of $x_{0}$ and $T>0$, where the neighborhood is open in the relative topology of the bottom stratum, $S_{B}$.

To clarify the presentation, we make one technical assumption. Later we will discuss the conditions necessary to eliminate this assumption.
Assumption 3.2 If $S_{I_{i+1}} \subset S_{I_{i}}$, in the gait, $\mathcal{G}=$ $\left\{S_{I_{1}}, S_{I_{2}}, \ldots, S_{I_{n}}, S_{I_{1}}\right\}$, then $S_{I_{i+1}}$ is a codimension one submanifold of $S_{I_{i}}$. In other words, multi-level "jumps" to lower level strata are not allowed.

Physically, this assumption requires that the robot put one foot down at a time instead of putting multiple feet down simultaneously.

Now, we construct the gait controllability distribution, which, in Proposition 3.3 will indicate whether the system is controllable. Recall that $\bar{\Delta}_{I}$ is the involutive closure of the distribution $\Delta_{I}$ defined by the vector fields which define the control system on stratum $S_{I}$. Given a gait, $\mathcal{G}$, the gait distribution defined on the bottom stratum, $S_{I_{1}}=S_{B}$, is the distribution

$$
\mathcal{D}_{m}=\sum_{i=2}^{m} \mathcal{D}_{i-1}+\left(\bar{\Delta}_{I_{i}} \cap T S_{B}\right)
$$

where $\mathcal{D}_{1}=\bar{\Delta}_{I_{1}}$.

The following Proposition is our main result.

\section{Proposition 3.3 If

$$
\operatorname{dim}\left(\mathcal{D}_{n}\right)=\operatorname{dim}\left(T_{x} S_{B}\right),
$$

then the system is gait controllable from $x$.

Proof: This proof is complete; however, a much more detailed version can be found in [10]. First, note that the dimension of the reachable set on any stratum, $S_{I_{i}}$ is equal to the dimension of $\bar{\Delta}_{I_{i}}$. This follows directly from Frobenius' Theorem (see, e.g., Theorem 4.4.7 of [11]), or from the standard proof of Chow's theorem (see, e.g., Proposition 3.15 of [12]).

To construct the reachable set on any stratum, $S_{i_{i}}$, consider the composition of flows of the form

$$
\mathcal{N}_{m}=\phi_{\epsilon_{m}}^{X_{m}} \circ \cdots \circ \phi_{\epsilon_{1}}^{X_{1}}\left(x_{0}\right),
$$

where $\phi_{t}^{X}$ is the flow along the vector field $X$ for time $t$ and $X_{i} \in \bar{\Delta}_{I_{i}}$. A variety of arguments (such as the orbit theorem, Theorem 1 of Chapter 2 of [13]) show that this is an $m$-dimensional submanifold of $S_{I_{i}}$. If $m<\operatorname{dim}\left(\bar{\Delta}_{I_{i}}\right)$, then there must be an $X_{m+1} \in \Delta_{I_{i}}$ such that

$$
\mathcal{N}_{m+1}=\phi_{\epsilon_{m+1}}^{X_{m+1}} \circ \phi_{\epsilon_{m}}^{X_{m}} \ldots \circ \phi_{\epsilon_{1}}^{X_{1}}\left(x_{0}\right),
$$

is an $m+1$-dimensional submanifold of $S_{I_{i}}$. If this were not the case, then $\bar{\Delta}_{I_{i}} \subset T S_{I_{i}}$, which is a contradiction since this would require that the dimension of $\bar{\Delta}_{I_{i}}$ be less than $m$. The reachable set is now constructed by extending it on a stratum-by-stratum basis through the gait.

LEMMA 3.4 In the construction of the reachable set, if $S_{I_{i}} \subset S_{I_{i+1}}$, then the dimension of the reachable 
set increases by the same amount as the increase in dimension between $\mathcal{D}_{i}$ and $\mathcal{D}_{i}+\bar{\Delta}_{I_{i+1}}$.

Proof: Let $\operatorname{dim}\left(\mathcal{D}_{I_{i}}\right)=m$ and $\operatorname{dim}\left(\mathcal{D}_{I_{i}}+\bar{\Delta}_{I_{i+1}}\right)=$ $m+n$, i.e., the dimension increases by $n$. By the definition of a gait, there exists a vector field $X_{\text {off }} \notin \mathcal{D}_{I_{\mathrm{i}}}$, that takes the system off of $S_{I_{i}}$ into $S_{I_{i+1}}$. Now, on $S_{I_{i+1}}$ by exactly the same argument as before, unless $\bar{\Delta}_{Y_{i+1}}$ is one dimensional, there must exist an $X_{m+1} \in \bar{\Delta}_{I_{i+1}} \subset \mathcal{D}_{I_{i+1}}$ such that

$$
\mathcal{N}_{m+1}=\phi_{\epsilon_{m+1}}^{X_{m+1}} \circ \phi_{\epsilon_{\circ f f}}^{X_{o f f}} \circ \phi_{\epsilon_{m}}^{X_{m}} \cdots \circ \phi_{\epsilon_{1}}^{X_{1}}\left(x_{0}\right),
$$

is an $m+2$-dimensional manifold. Repeating this same argument for the existence of $X_{m+2}, X_{m+3}, \ldots, X_{m+n}$ gives the reachable set $\mathcal{N}_{m+1}=\phi_{\epsilon_{m+n}}^{X_{m+n}} \circ \cdots \circ \phi_{\epsilon_{o f f}}^{X_{o f f}} \ldots \circ$ $\phi_{\epsilon_{1}}^{X_{1}}\left(x_{0}\right)$, which is an $m+n$-dimensional manifold.

LEMMA 3.5 In the construction of the reachable set, if $S_{I_{i+1}} \subset S_{I_{i}}$, then the dimension of the reachable set increases by the same amount as the increase in dimension between $\mathcal{D}_{I_{i}}$ and $\mathcal{D}_{I_{i}}+\bar{\Delta}_{I_{i+1}}$ minus the difference between the dimensions of $S_{I_{i+1}}$ and $S_{I_{1}}$.

Proof: First, the reachable set must be restricted to the submanifold $S_{I_{i+1}}$. However, Assumption $3.2 \mathrm{im}-$ plies that $S_{I_{i}}$ and $S_{I_{i+1}}$ intersect transversely. This is because $S_{I_{i+1}}$ is a codimension 1 submanifold of the $M$, and, by the definition of a gait, the reachable set on $S_{I_{i}}$ intersects it. The standard result for transverse submanifolds (Corollary 3.5.13 of [11]),

$$
\operatorname{codim}\left(S_{I_{i}} \cap S_{I_{i+1}}\right)=\operatorname{codim}\left(S_{I_{i}}\right)+\operatorname{codim}\left(S_{I_{i+1}}\right),
$$

implies that the dimension of the reachable set decreases by one. Now, the increase in dimension due to the sum of $\mathcal{D}_{I_{i}}$ and $\mathcal{D}_{I_{i+1}}$ (restricted to $S_{I_{i+1}}$ ), follows, again, from the argument before and in the previous lemma.

It follows that in the construction of the gait distribution that the dimension of the reachable set will be the dimension of $\mathcal{D}_{n}$. If the first and last strata in the gait $\mathcal{G}$ is the bottom stratum, then the result follows since the reachable set it contained in $S_{B}$ and has dimension equal to the dimension of $S_{B}$.

We assumed that if $S_{I_{i+1}} \subset S_{I_{i}}$, then the reachable set is transversal to the substratum, $S_{I_{i+1}}$. As noted, this is natural if $\operatorname{dim}\left(S_{I_{i+1}}\right)=\operatorname{dim}\left(S_{I_{i}}\right)-1$, which was assumed by Assumption 3.2. This assumption can be relaxed to allow switches between strata with dimensions which vary by more than one as long as this transversality assumption is satisfied.

\section{An Example}

The following example is adapted from Kelly and Murray [7]. Consider the six-legged robot shown in
Figure 1. Recall, as discussed in the introduction, it is not obvious that the robot can move in any direction.

Assume that the robot walks with a tripod gait, alternating movements of legs $1-4-5$ with movements of legs $2-3-6$. The equations of motion are

$$
\begin{aligned}
\dot{x} & =\cos \theta\left(\alpha\left(h_{1}\right) u^{1}+\beta\left(h_{2}\right) u^{2}\right) \\
\dot{y} & =\sin \theta\left(\alpha\left(h_{1}\right) u^{1}+\beta\left(h_{2}\right) u^{2}\right) \\
\dot{\theta} & =l \alpha\left(h_{1}\right) u^{1}-l \beta\left(h_{2}\right) u^{2} \\
\dot{\phi}_{1} & =u^{1} \quad \dot{h}_{1}=u^{3} \\
\dot{\phi}_{2} & =u^{2} \quad \dot{h}_{2}=u^{4}
\end{aligned}
$$

where $(x, y, \theta)$ represents the planar position of the center of mass, $\phi_{i}$ is the front to back angular deflection of the legs, $h_{i}$ is the height of the legs off the ground and $l$ relates the rotation of the body to the leg deflection. The tripod gait assumption requires that all the legs in a tripod move with the same velocity $\dot{\phi}_{1}=\dot{\phi}_{4}=\dot{\phi}_{5}$ and $\dot{h}_{1}=\dot{h}_{4}=\dot{h}_{5}$, and similarly for legs 2-3-6. The inputs $u^{1}$ and $u^{2}$ control the leg swing velocities, while the inputs $u^{3}$ and $u^{4}$ control the leg lifting velocities.

The functions $\alpha\left(h_{1}\right)$ and $\beta\left(h_{2}\right)$ are defined by

$$
\alpha\left(h_{1}\right)=\left\{\begin{array}{ll}
1 & \text { if } h_{1}=0 \\
0 & \text { if } h_{1}>0
\end{array} \quad \beta\left(h_{2}\right)=\left\{\begin{array}{ll}
1 & \text { if } h_{2}=0 \\
0 & \text { if } h_{2}>0
\end{array} .\right.\right.
$$

If all legs are in contact with the ground $\left(S_{123456}\right)$, the equations of motion are

$$
\left(\begin{array}{c}
\dot{x} \\
\dot{y} \\
\dot{\theta} \\
\dot{\phi}_{1} \\
\dot{\phi}_{2}
\end{array}\right)=\left(\begin{array}{cccc}
\cos \theta & \cos \theta & 0 & 0 \\
\sin \theta & \sin \theta & 0 & 0 \\
l & -l & 0 & 0 \\
1 & 0 & 0 & 0 \\
0 & 1 & 0 & 0
\end{array}\right)\left(\begin{array}{l}
u^{1} \\
u^{2} \\
u^{3} \\
u^{4}
\end{array}\right)
$$

where $u^{3}$ and $u^{4}$ are constrained to be 0 . Note that if we let $f$ represent the first column, and $g$ the second column, then

$$
[f, g]=\left(\begin{array}{c}
-2 l \sin \theta \\
2 l \cos \theta \\
0 \\
0 \\
0
\end{array}\right) .
$$

Clearly, on $S_{123456}$, we have generated enough directions to span the $(x, y, \theta)$ directions, but not enough to span all the shape variables (the $h_{i}$ and $\phi_{i}$ directions) as well.

On $S_{145}$ the equations of motion are

$$
\left(\begin{array}{c}
\dot{x} \\
\dot{y} \\
\dot{\theta} \\
\dot{\phi}_{1} \\
\dot{\phi}_{2} \\
\dot{h}_{2}
\end{array}\right)=\left(\begin{array}{cccc}
\cos \theta & 0 & 0 & 0 \\
\sin \theta & 0 & 0 & 0 \\
l & 0 & 0 & 0 \\
1 & 0 & 0 & 0 \\
0 & 1 & 0 & 0 \\
0 & 0 & 0 & 1
\end{array}\right)\left(\begin{array}{l}
u^{1} \\
u^{2} \\
u^{3} \\
u^{4}
\end{array}\right)
$$


where $u^{3}$ is constrained to be 0 .

On $S_{236}$ the equations of motion are

$$
\left(\begin{array}{c}
\dot{x} \\
\dot{y} \\
\dot{\theta} \\
\dot{\phi}_{1} \\
\dot{\phi}_{2} \\
\dot{h}_{1}
\end{array}\right)=\left(\begin{array}{cccc}
0 & \cos \theta & 0 & 0 \\
0 & \sin \theta & 0 & 0 \\
0 & -l & 0 & 0 \\
1 & 0 & 0 & 0 \\
0 & 1 & 0 & 0 \\
0 & 0 & 1 & 0
\end{array}\right)\left(\begin{array}{l}
u^{1} \\
u^{2} \\
u^{3} \\
u^{4}
\end{array}\right)
$$

where $u^{4}$ is constrained to be 0 .

Now, we must construct the gait distribution. We take as our gait, the following sequence of strata:

$$
\mathcal{G}=\left\{S_{123456}, S_{145}, S_{123456}, S_{236}, S_{123456}\right\},
$$

as illustrated in Figure 4. The equations of motion for the system restricted to the bottom stratum, $S_{123456}$ are given in Equation 5. Also, a Lie bracket is necessary to construct $\bar{\Delta}_{123456}$, as given in Equation 6 . By inspection, $\bar{\Delta}_{123456}=\mathcal{D}_{1}$ has a dimension of three.

Now extend the construction to $S_{145}$. Since $S_{123456} \subset S_{145}, \mathcal{D}_{2}=\mathcal{D}_{1}+\bar{\Delta}_{145}$, where $\bar{\Delta}_{145}$ is determined from Equation 7. By inspection, then, $\operatorname{dim}\left(\mathcal{D}_{2}\right)=5$.

Next, the construction returns to the bottom stratum, $S_{123456}$. We note that $S_{12}$ is a codimension 1 submanifold of $S_{1}$. Also, since $\mathcal{D}_{2}$ contains the basis vector $\frac{\partial}{\partial h_{1}}$, Assumption 3.2 holds. Then, we have that $\mathcal{D}_{3}=\mathcal{D}_{2}-1=4$.

Now, the construction is extended to stratum $S_{236}$. As with $S_{145}, S_{236}$ increases the dimension of $\mathcal{D}_{4}$ by two, so that $\operatorname{dim}\left(\mathcal{D}_{4}\right)=6$. "Projecting" this back down to $S_{12}$ as before gives the dimension of the reachable set to be 5 , which is the dimension of $S_{123456}$. Therefore, the hexapod example is gait controllable.

\section{Conclusions}

This paper presented a general method to determine whether a specific gait of a legged robot is controllable. One attractive feature of this method is that it is independent of the particular morphology of the robot, and, more specifically, independent of the number of legs. This is a consequence of the fact that this method is an extension of general nonlinear control techniques, which, themselves are of general applicability for smooth systems. Also, the test has applicability beyond problems of legged locomotion. Grasping is one obvious related problem in which the finger gaiting (lifting and replacing fingers) is the analog of walking, and the question of controllability relates to whether or not it is possible to reorient a grasped object to any arbitrary orientation.

The main limitation of this work, and thus a prospect for future work, is the limitation of the method to driftless systems, or quasi-static robotic lo- comotors. Eliminating this limitation would make the method applicable to an even broader class of legged robotic systems, including dynamic bipeds and hopping monopods. The main difficulty is that, even for smooth systems, the "state of the art" for systems with drift is much less developed. A general theorem on local controllability for system with drift does exist [14]; however, it only provides sufficient conditions for controllability. Even more limiting is that it is only valid at equilibrium points, which further inhibit an extension of the theory to stratified systems.

\section{Acknowledgments}

This research was partially supported by the Office of Naval Research.

\section{References}

[1] S. Kajita and K. Tani. Study of dynamic biped locomotion on rugged terrain. In Proc. IEEE Int. Conf. Robotics and Automation, pages 1405-1411, Sacramento, CA, April 1991.

[2] J.K. Lee and S.M. Song. Path planning and gait of walking machine in an obstacle-strewn environment. J. Robotics Systems, 8:801-827, 1991.

[3] S.M. Song. and K.J. Waldron. Machines that walk: the Adaptive Suspension Vehicle. MIT Press, 1989.

[4] M.H. Raibert. Legged Robots that Balance. MIT Press, 1986.

[5] Bill Goodwine and Joel Burdick. Trajectory generation for kinematic legged robots. In IEEE International Conference on Robotics and Automation, pages 2689-2696. IEEE, 1997.

[6] J. William Goodwine. Control of Stratified Systems with Robotic Applications. PhD thesis, California Institute of Technology, 1998.

[7] Scott D. Kelly and Richard M. Murray. Geometric phases and robotic locomotion. J. Robotic Systems, 12(6):417-431, 1995.

[8] J. Ostrowski, J. Burdick, R. Murray, and A. Lewis. The mechanics of undulatory locomotion: the mixed dynamic and kinematic case. In IEEE Int. Conf. on Robotics and Automation, Nagoya, Japan, May 1995.

[9] J.P. Ostrowski. The Mechanics and Control of Undulatory Robotic Locomotion. PhD thesis, California Institute of Technology, Pasadena, CA, Sept. 1995.

[10] Bill Goodwine and Joel Burdick. Controllability of kinematic systems on stratified configuration spaces. In preparation, available electronically at http://robby.caltech.edu/papers.html.

[11] R. Abraham, J. E. Marsden, and T. Ratiu. Manifolds, Tensor Analysis, and Applications. Springer-Verlag, second edition, 1988.

[12] H. Nijmeijer and A. J. Van der Schaft. Nonlinear Dynamical Control Systems. Springer-Verlag, 1990.

[13] Velimir Jurdjevic. Geometric Control Theory. Cambridge University Press, 1997.

[14] Hector J. Sussmann. A general theorem on local controllability. Siam J. Control and Optimization, 25(1):158-194, 1987. 